\title{
Measurements of the transverse emittance at the FLASH injector at DESY
}

\author{
F. Löhl* and S. Schreiber \\ Deutsches Elektronen Synchrotron (DESY), Notkestraße 85, 22607 Hamburg, Germany \\ M. Castellano and G. Di Pirro \\ INFN-Laboratori Nazionali di Frascati, Via E. Fermi,40, 00044 Frascati (Roma), Italy \\ L. Catani and A. Cianchi \\ INFN Sezione di Roma Tor Vergata, Via della Ricerca Scientifica, 1, 00133 Roma, Italy
}

K. Honkavaara

Hamburg University, Institut für Experimentalphysik, Luruper Chaussee 149, 22761 Hamburg, Germany

(Received 17 May 2006; published 18 September 2006)

\begin{abstract}
The VUV and soft x-ray free electron laser FLASH (former VUV-FEL) is a user facility at DESY (Hamburg). In order to optimize the performance of the facility, an accurate characterization of the electron beam properties is essential. The transverse projected emittance, one of the important parameters characterizing the quality of an electron beam, is measured using a four monitor method with optical transition radiation monitors. A normalized rms emittance below $2 \mathrm{~mm}$ mrad for a $1 \mathrm{nC}$ beam has been measured. In this paper we describe the experimental setup, data analysis methods, and present experimental results.
\end{abstract}

DOI: 10.1103/PhysRevSTAB.9.092802

PACS numbers: 29.27.Fh

\section{INTRODUCTION}

The vacuum ultraviolet free electron laser (VUV-FEL) [1], now FLASH (Free electron LASer in Hamburg), is a FEL user facility at DESY (Hamburg). It is based on the SASE (Self-Amplified Spontaneous Emission) process [2] to produce FEL radiation in the wavelength range from vacuum ultraviolet to soft $\mathrm{x}$ rays. The commissioning of the facility started in the beginning of 2004 and the first lasing with a wavelength of $32 \mathrm{~nm}$ was achieved in January of 2005 [3]. User experiments started in the summer of 2005.

Figure 1 shows the present layout of the linac. Electron bunch trains with a bunch charge of $1 \mathrm{nC}$ are generated by a laser driven rf gun [4]. Five accelerating modules with eight 9-cell superconducting niobium cavities [5] each are installed to provide an electron beam energy up to $700 \mathrm{MeV}$. Later, one additional accelerating module will be added to increase the electron beam energy to $1 \mathrm{GeV}$. The electron bunch is compressed using two magnetic chicane bunch compressors. At the location of the first bunch compressor the beam energy is $125-130 \mathrm{MeV}$, and at the second one about $380 \mathrm{MeV}$. During commissioning, the main emphasis was on lasing with a photon wavelength of $32 \mathrm{~nm}$, corresponding to an electron beam energy of $445 \mathrm{MeV}$.

A high quality electron beam is required for the lasing process. At FLASH the design value of the normalized transverse emittance is $2 \mathrm{~mm}$ mrad, the design peak current $2500 \mathrm{~A}$, and the design energy spread $0.1 \%$. In this paper

*Electronic address: florian.loehl@desy.de we present measurements of the transverse projected emittance at the injector with a beam energy of $\sim 130 \mathrm{MeV}$. The measurements are performed in a special diagnostic section (Fig. 1) using a four monitor method with optical transition radiation (OTR) monitors.

\section{EXPERIMENTAL SETUP}

The injector consists of a laser driven rf gun with a solenoid magnet to counteract space charge induced emittance growth, a booster cavity to increase the electron beam energy up to $125-130 \mathrm{MeV}$, a bunch compressor, and a diagnostic section for measurements of the transverse emittance. More details of the injector concept, the present stage of the injector, as well as the planned future upgrades are in [6].

The diagnostic section located downstream of the first bunch compressor consists of six quadrupoles with alternating polarity and four OTR beam profile monitors combined with wire scanners. In this paper we concentrate on emittance measurements using the OTR monitors only.

\section{A. OTR monitors}

Transition radiation is electromagnetic radiation emitted when a charged particle crosses a boundary between two media of different optical properties, in practice a thin mirror like wafer. Optical transition radiation (OTR), the visible part of this radiation, provides a fast single shot measurement of the transverse charge distribution of an electron beam.

The requirements for the OTR monitors are demanding: besides delivering on-line beam images, they are used to 

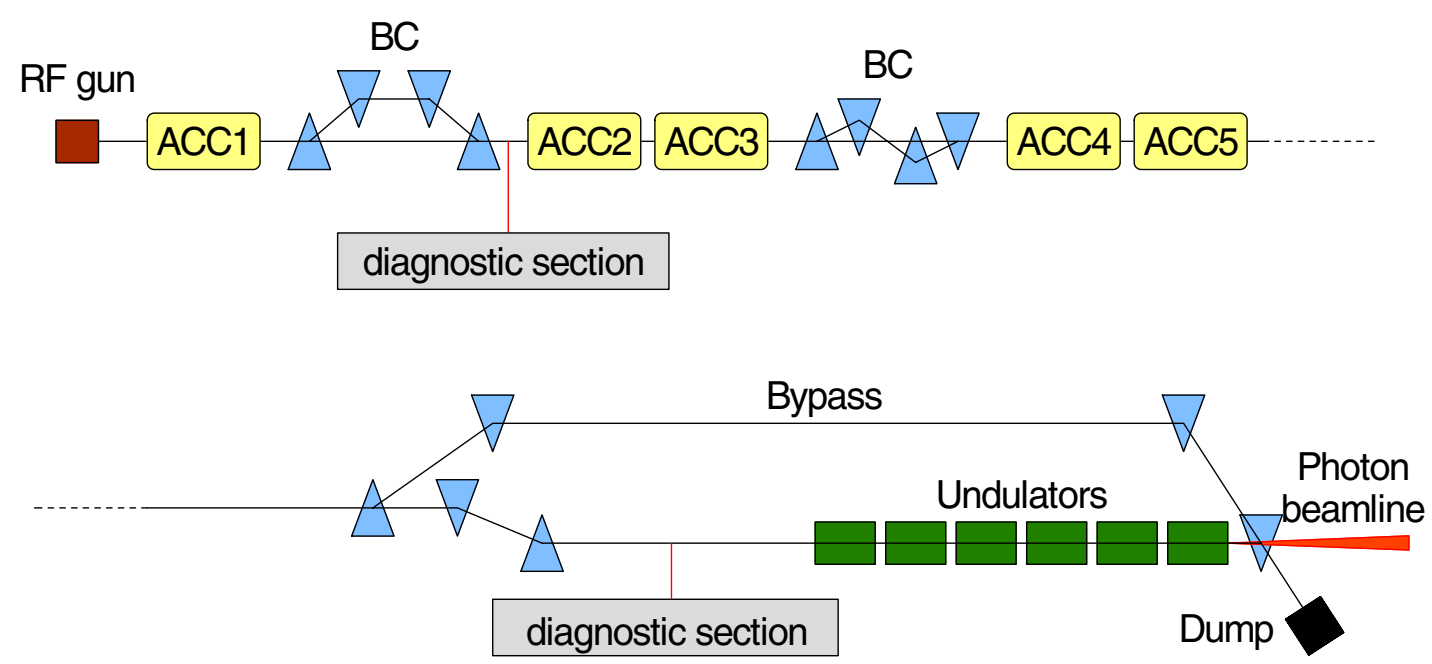

FIG. 1. (Color) Present layout of the linac (not to scale). Beam direction is from left to right. The electron bunches are accelerated in the superconducting structures ACC1-ACC5 and compressed longitudinally in two magnetic chicane bunch compressors. The total length is about $250 \mathrm{~m}$. The emittance measurements discussed in this paper are performed in the diagnostic section after the first bunch compressor.

measure transverse beam sizes from millimeter scale down to $\sim 50 \mu \mathrm{m}$ with high resolution. In addition, the complete system has to be reliable, robust, and remote controlled. The OTR system has been designed and constructed by INFN-LNF and INFN-Roma Tor Vergata in collaboration with DESY. These monitors measure backward OTR emitted when the electron beam traverses the screen. They have two screens, a polished silicon screen (higher damage threshold) and a silicon screen with an aluminum coating (better image quality), mounted at an angle of $45^{\circ}$ with respect to the beam trajectory. The screens are inserted into the beam pipe by a stepper motor actuator. In order to calibrate and adjust the optical system, a calibration target can be inserted.

The optical system includes three achromat doublet lenses, three neutral density filters, and a CCD camera. The lenses and filters can be moved remotely in and out of the optical axis of the system. Each of the lenses provides a fixed magnification: $1.0,0.39$, or 0.25 . In order to avoid a decrease of the resolution due to aberrations, a diaphragm with a diameter of $20 \mathrm{~mm}$ is mounted on the lens with the highest magnification (1.0). The measured resolution for the 1.0 magnification is $11 \mu \mathrm{m} \mathrm{rms}$. A design emittance of $2 \mathrm{~mm}$ mrad corresponds to rms beam sizes in the diagnostic section of about $140 \mu \mathrm{m}$. The optical setups are prealigned and calibrated in a laboratory prior to installation. The complete system is covered from external light, and the CCD camera has a lead shield against radiation. More details of the mechanical construction and performance of the system are in $[7,8]$.

The readout system is based on the use of digital CCD cameras with a firewire interface (IEEE1394). The cameras (totally $\sim 30$ along the linac) are connected via firewire links to compact industrial PCs (totally 8) located in the accelerator tunnel. The PCs are connected via local
Ethernet to an "image server" in the control room. The image server, using LabView based control and image analysis software, provides the main interface for the whole camera system. House-made remotely controlled power switches allow one to reset the PCs and the cameras from the control room. More details of the readout system can be found in [9].

\section{DATA ANALYSIS}

The four (multi)monitor method is based on measurements of the transverse beam distribution at four (or more) locations with a fixed beam optics. The normalized rms emittance is defined as

$$
\epsilon_{\mathrm{N}, \mathrm{u}}=\frac{p}{m_{0} c} \sqrt{\left\langle u^{2}\right\rangle\left\langle u^{\prime 2}\right\rangle-\left\langle u u^{\prime}\right\rangle^{2}}, \quad u=x, y .
$$

It is determined from the measured beam distributions and the known transfer matrices between the monitors using two different methods. The first one is a least square fitting of the Twiss parameters and the emittance to the measured beam sizes (see e.g. [10]). The second method is based on a tomographic reconstruction of the phase space distribution using the maximum entropy algorithm (MENT) [11].

\section{A. Image analysis}

For a standard emittance measurement we record 20 beam and background images at each of the four OTR screens. In order to reduce the influence of dark current and possible damaged camera pixels as well as to correct offsets generated by, for example, noise in the camera system, an averaged background image is subtracted from each beam image. Even with the highest magnification of the OTR monitors, a measured beam distribution usually covers only a small part of the entire image. It is, therefore, 
advantageous to evaluate only the small part of the image which includes the beam. Using such a region of interest (ROI), the signal to noise ratio of the measured beam distribution is increased and in addition we get the possibility to determine offsets with high accuracy from the remaining part of the image outside the ROI.

Figure 2 shows the dependence of the calculated beam sizes on the size of the ROI for a measured beam distribution. After subtracting the averaged background image, we choose as a starting ROI a circle with a radius of 10 pixels. The maximum of the beam profile in the horizontal and vertical plane is defined as the center. The part of the image outside of the ROI is used to calculate an average value for the offset which then is subtracted from the entire image. For the calculation of the rms beam sizes, only the fraction of the image inside the ROI is used. The next regions of interest, indicated by an index $i$ in Eq. (2), are ellipses that are chosen such that the semiaxis in the $x$-direction, $a_{x}$, increases continuously while the semiaxis in the $y$-direction, $a_{y}$, is scaled with the ratio of the rms beam sizes calculated for the previous, smaller ROI, indicated by the index $i-1$ :

$$
a_{y}^{(i)}=a_{x}^{(i)} \frac{y_{\mathrm{rms}}^{(i-1)}}{x_{\mathrm{rms}}^{(i-1)}}=a_{x}^{(i)} \frac{\sqrt{\left\langle y_{(i-1)}^{2}\right\rangle}}{\sqrt{\left\langle x_{(i-1)}^{2}\right\rangle}} .
$$

In each step, a different offset resulting from the average intensity outside the ROI is subtracted from the entire image.

Increasing the size of the ellipse for a small ROI that does not contain the entire beam results in a steep increase of the rms beam sizes (see Fig. 2). At a certain ellipse size,

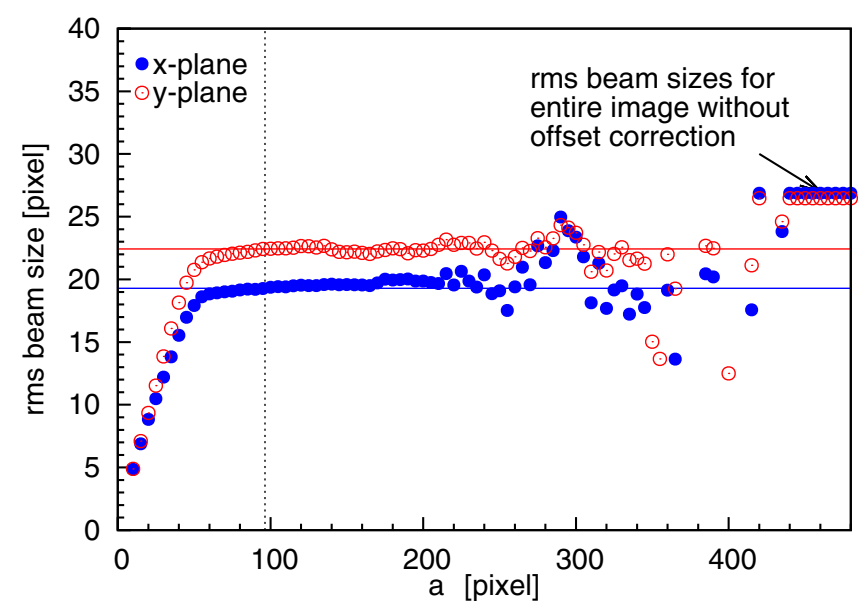

FIG. 2. (Color) Dependence of the reconstructed beam sizes on the size of the region of interest. Plotted is the horizontal semiaxis $a_{x}$ (see text). The dotted line shows the horizontal semiaxis $a_{x}$ of the ellipse resulting from the algorithm used for the analysis of the beam images. the determined rms beam sizes become insensitive to small changes in the ellipse size. The ROI contains now the entire beam and also the offset is well estimated. Further increase of the ellipse size results again in instable rms beam sizes. The reason for this behavior is that in the case of large regions of interest only a few pixels are left over to calculate the offset. Small fluctuations in these pixels, therefore, have strong influence on the rms beam sizes. For the analysis of the beam images, we use an algorithm which converges in the stable region (dotted line in Fig. 2). Since no threshold procedures are applied, this method allows us to reconstruct also the beam tails with high precision.

A small fraction of particles in the tails of the distribution can have a significant influence on the measured emittance. Therefore, in addition to the emittance of the entire beam, we are also interested in the emittance of the high density core. This core is determined by cutting away $10 \%$ (an arbitrary choice) of particles in the tails of the transverse beam distribution. After that the horizontal and vertical rms beam sizes of the core containing $90 \%$ of the beam intensity are calculated.

The rms beam sizes defined as above are used to calculate the emittance and the Twiss parameters with a least square fit resulting in the rms emittance of the entire beam and the rms emittance of the beam core including $90 \%$ of the particles.

In the tomographic reconstruction an averaged beam profile of the entire beam for each screen is used. The power of the MENT algorithm to reconstruct twodimensional density distributions from only a few profiles is demonstrated in [12]. In order to avoid broadening of the profile due to a beam position jitter, the measured profiles are rebinned and the center of each profile is moved to the same position before averaging. The emittance of the entire beam is then determined by the maximum entropy algorithm. In order to obtain the $90 \%$ core emittance, $10 \%$ of the particles in the tails of the reconstructed phase space distribution are cut away.

For more details about the image analysis see [12].

\section{B. Systematic errors and error analysis}

The dependence of the systematic error of the normalized emittance on the choice of the optics inside the FODO lattice has been studied. Figure 3 shows the systematic error in the normalized emittance as a function of the Twiss parameters $\alpha$ and $\beta$ at the first OTR screen in the FODO lattice for a 5\% error in the beam sizes (top), and in the measured beam energy (bottom). In Fig. 4 a cut through the two-dimensional graphs of Fig. 3 is shown. The error in the normalized emittance for an error in the beam sizes is evaluated from the covariance matrix of the $\chi$-square fit. To determine the dependence of the normalized emittance on beam energy errors, the beam energy used in the calculations was assumed to be $5 \%$ higher than the real one. This deviation was chosen since the present layout of the 


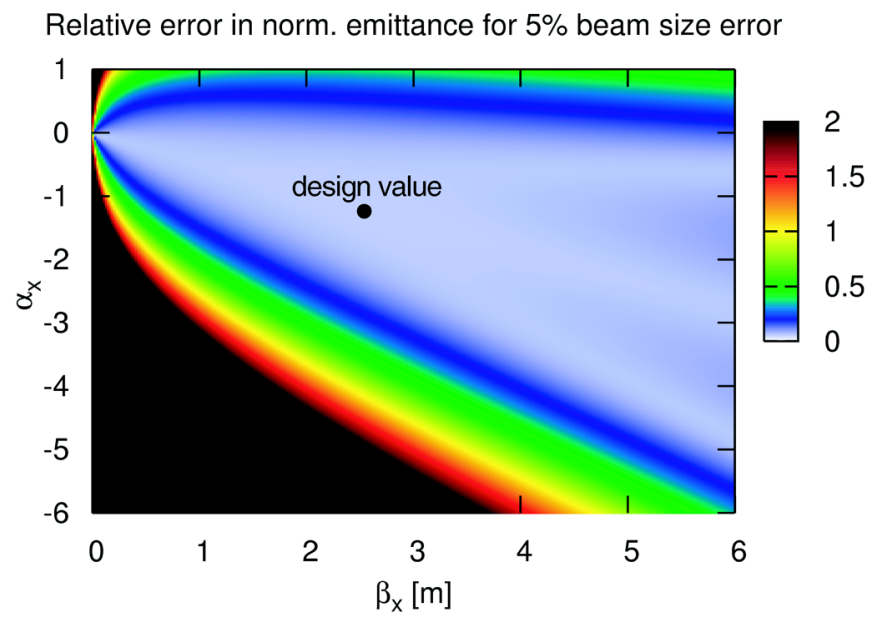

Relative error in norm. emittance for an energy error of $+5 \%$

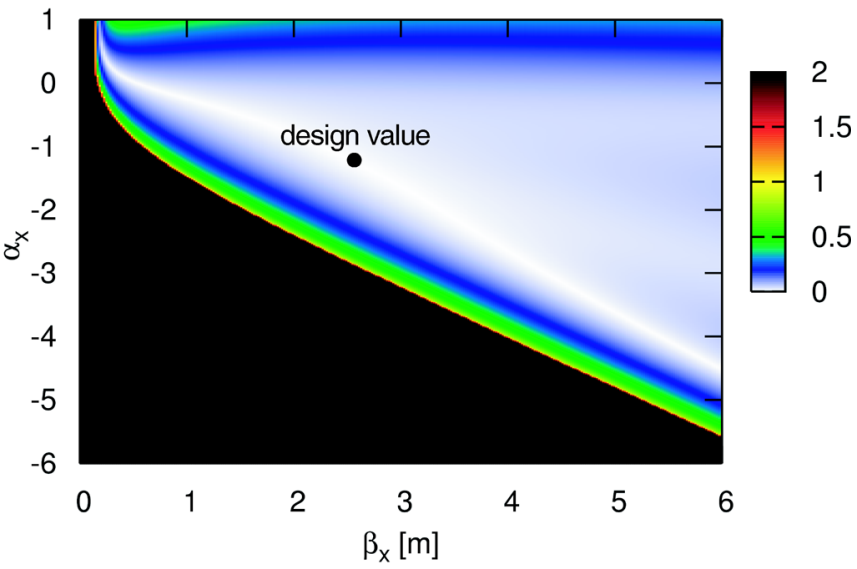

FIG. 3. (Color) Relative systematic error in the normalized horizontal emittance as a function of the Twiss parameters $\alpha$ and $\beta$ at the first screen in the FODO lattice for a 5\% error in the beam sizes (top) and 5\% error in the beam energy (bottom). The color black indicates a relative error in the normalized emittance larger than $200 \%$.

bunch compressor section does not allow us to measure the absolute beam energy better than $3 \%$ to $5 \%$. Figures 3 and 4 indicate that for accurate emittance measurements a correctly matched beam inside the FODO lattice is essential. In the measurements presented here, we have always taken care that the Twiss parameters inside the FODO lattice are matched to the design values.

The error estimation for the emittance determination using the fitting technique has been performed taking into account both statistical and systematic errors. Statistical errors caused by fluctuations of the measured beam sizes are calculated as in [10]. Systematic errors are estimated using a Monte Carlo simulation assuming 5\% error in the beam energy, $6 \%$ error in the gradient of the FODO lattice quadrupoles, and 3\% error in the calibration of the optical system. Statistical errors are typically $2 \%-4 \%$ and systematic ones $5 \%-6 \%$.

More details of the error analysis are in [12].
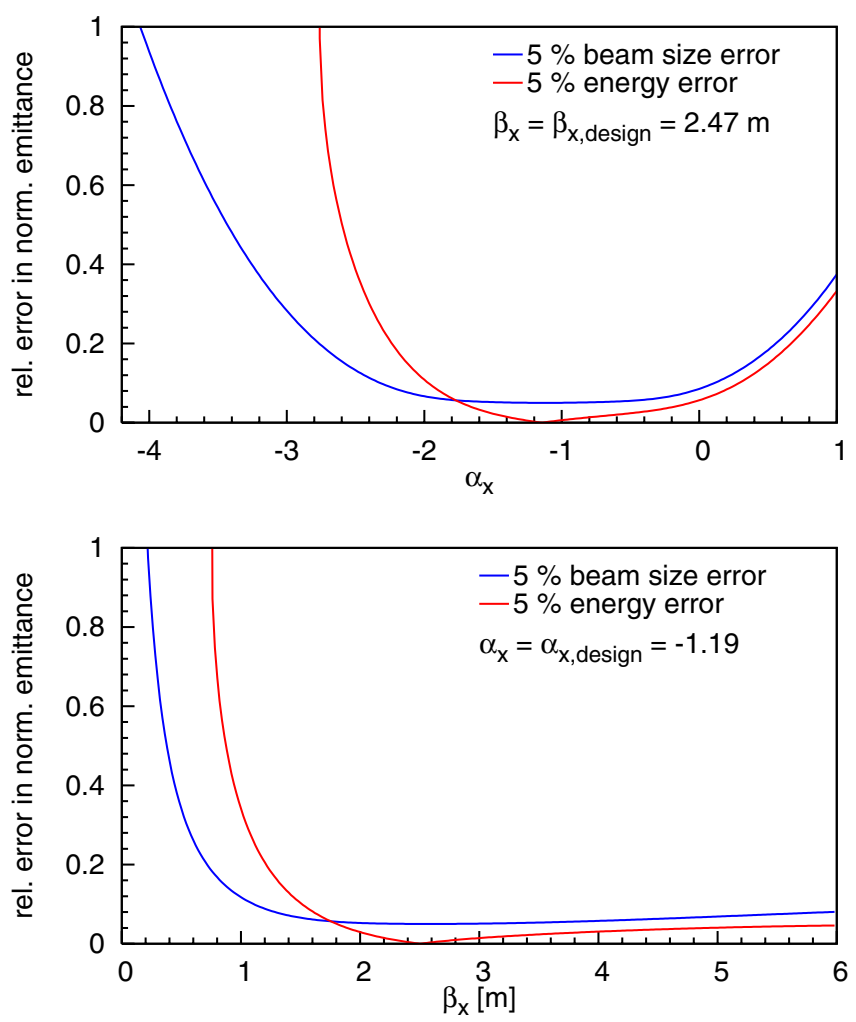

FIG. 4. (Color) Cut through the two-dimensional diagrams depicted in Fig. 3 showing the dependence of the relative systematic error in the normalized emittance as a function of $\alpha_{x}$ for $\beta=\beta_{x \text {,design }}$ (top) and as a function of $\beta_{x}$ for $\alpha=\alpha_{x \text {,design }}$ (bottom).

\section{MEASUREMENTS AND RESULTS}

The measurements presented here are performed with nominal injector parameters close to the parameters determined during precommissioning of the rf gun at PITZ [13]: an electron bunch charge of $1 \mathrm{nC}, 3 \mathrm{MW}$ rf power in the rf gun, a phase of 30-40 deg between the rf gun and the laser, and a beam energy of 125-127 MeV after acceleration by the first accelerating module. The laser beam profile is longitudinally Gaussian with a length of 10 ps (FWHM). The transverse laser profile is a Gaussian with $\sigma_{x, y}=$ $1 \mathrm{~mm}$, truncated by a $3.5 \mathrm{~mm}$ diameter iris. The photocathode has a diameter of $5 \mathrm{~mm}$. The electron beam is transported through the first bunch compressor without bunch compression (on-crest acceleration in the accelerating module). Some experimental results presented here have also been discussed in [14-16].

Figure 5 shows the normalized horizontal and vertical rms emittances measured 10 times within $1.5 \mathrm{~h}$ without changing the machine parameters. The results obtained by the fitting technique and by the phase space tomography are presented for $100 \%$ and $90 \%$ beam intensity (fit $100 \%$ and $90 \%$, MENT $100 \%$ and $90 \%$, respectively). The results obtained by the different analysis techniques agree well 

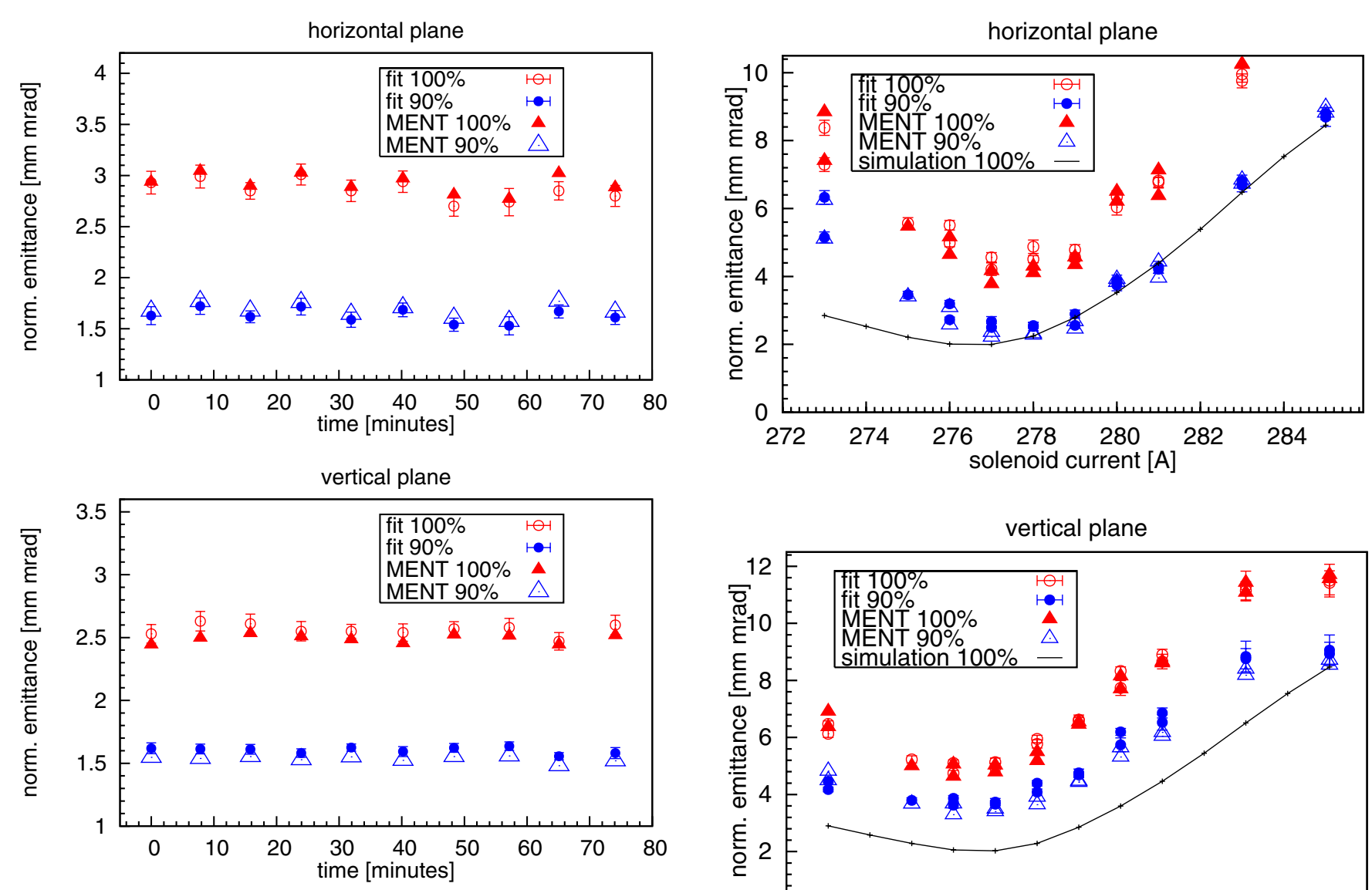

FIG. 5. (Color) Horizontal (top) and vertical (bottom) normalized rms emittances. The measurement is repeated 10 times during $\sim 1.5 \mathrm{~h}$. Results obtained by fitting for $100 \%$ (red circle) and $90 \%$ (blue circle) beam intensity as well as by tomography (MENT) for $100 \%$ (red triangle) and 90\% (blue triangle) intensity are shown. Error is the statistical error only.

with each other, and the stability of the measurements is good. The rms jitter of the $100 \%$ emittance in the horizontal plane is $\sim 3.5 \%$ and in the vertical $\sim 2 \%$, in agreement with the statistical error estimated above.

In order to reduce space charge induced emittance growth, the electron beam is focused by a solenoid magnet. Figure 6 shows the normalized horizontal and vertical rms emittances as a function of the current of this solenoid. For each solenoid current the beam has been rematched to the FODO lattice. Then the emittance is measured twice for each solenoid current. The result from simulations [17] using a normalized projected emittance of $2 \mathrm{~mm}$ mrad at the optimal working point for the solenoid is shown as a solid line. We can see that the behavior as a function of the solenoid current predicted by the simulations agrees well with the measurements. The optimal solenoid current, from the emittance point of view, is around $277 \mathrm{~A}$, which corresponds to a magnetic field of $0.163 \mathrm{~T}$. One should note that the optimal solenoid current depends on the used rf-gun input power. The measurements presented here are

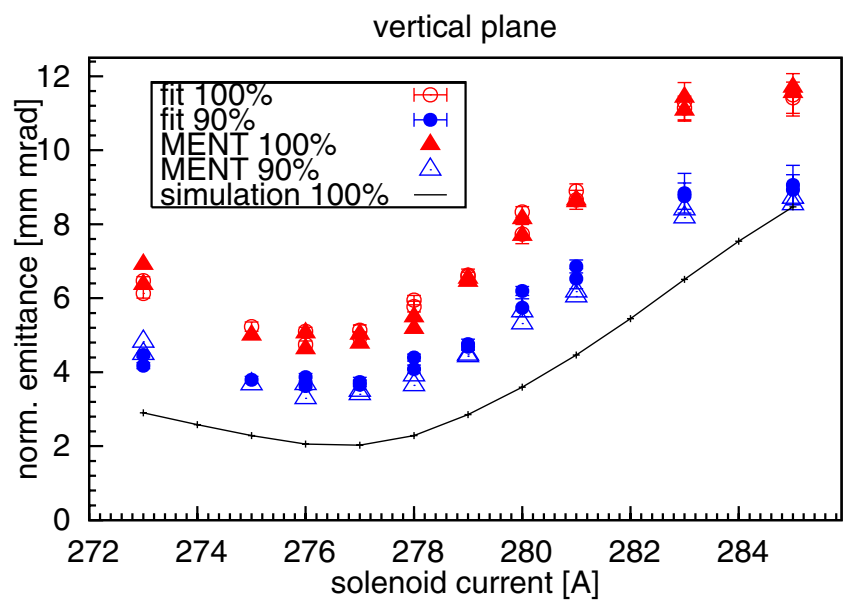

FIG. 6. (Color) Horizontal (top) and vertical (bottom) normalized rms emittance measured as a function of the solenoid current. Results obtained by fitting for $100 \%$ (red circle) and $90 \%$ (blue circle) beam intensity as well as by tomography (MENT) for $100 \%$ (red triangle) and $90 \%$ (blue triangle) intensity are shown. Error is the statistical error only. The solid line is not a fit but a prediction from simulations assuming the design emittance of $2 \mathrm{~mm}$ mrad.

performed with a power of about $3 \mathrm{MW}$. With a larger input power, the optimal solenoid current is correspondingly larger. A small remnant magnetic field on the cathode is zeroed by a bucking coil.

During the measurements presented above, the injector was operated with the nominal settings, but since the solenoid scan is a first step of the optimizing process, it was not yet tuned to obtain the minimum emittance. In order to achieve the smallest emittance, it is important to optimize, in addition to the solenoid, rf, and laser settings, also the beam injection to the first accelerating module. When the injector is carefully tuned and the beam is correctly steered through the accelerating module, we have regularly measured normalized rms emittances around $1.4 \mathrm{~mm}$ mrad for $90 \%$ of a $1 \mathrm{nC}$ bunch. For the entire bunch, this value is typically around $2 \mathrm{~mm}$ mrad. 


\section{SUMMARY AND OUTLOOK}

The emittance measurement system based on a four monitor method using OTR monitors is routinely used to measure projected emittances at the FLASH injector. We have regularly measured normalized rms emittances below $2 \mathrm{~mm}$ mrad for a $1 \mathrm{nC}$ bunch at $127 \mathrm{MeV}$ beam energy. A sophisticated image analysis algorithm has been developed for these measurements. Including data acquisition, image analysis and emittance calculation a measurement takes less than $5 \mathrm{~min}$.

In addition to the diagnostic section in the injector, a similar section consisting of a FODO lattice with four OTR monitors is located after all accelerating modules upstream of the undulator. However, due to present priorities of the accelerator operating as a FEL user facility, this section has not yet been commissioned for accurate emittance measurements. Along the linac it is possible to measure the emittance at several locations using a quadrupole scan technique. Since these measurements are time consuming and require typically a special beam optics, they are rarely performed. Along the undulator the emittance can be measured by a multimonitor technique using wire scanners mounted between the undulator modules. We plan to optimize the emittance measurement conditions along the linac and to measure the emittance simultaneously at several locations in order to gather information about the emittance transport.

\section{ACKNOWLEDGMENTS}

We thank the colleagues and the technical staff at DESY, at INFN-LNF, and at INFN-Roma Tor Vergata for contributing in the design, realization, and maintenance of the OTR monitor system. Special thanks go to the shift crews for participating in the emittance measurements, and to Y. Kim for providing the simulation results. For fruitful discussions on optics and on handling of systematic errors we like to thank V. Balandin.

[1] Valeri Ayvazyan et al. (The TTF FEL Team), TESLA-FEL 2002-01 (2002).

[2] E. L. Saldin, E. A. Schneidmiller, and M. V. Yurkov, The Physiscs of Free Electron Lasers (Springer, Berlin, 1999).

[3] V. Ayvazyan, N. Baboi, J. Bähr, V. Balandin, B. Beutner, A. Brandt, I. Bohnet, A. Bolzmann, R. Brinkmann, O. I. Brovko, J. P. Carneiro, S. Casalbuoni, M. Castellano, P. Castro, L. Catani, E. Chiadroni, S. Choroba, A. Cianchi, H. Delsim-Hashemi, G. Di Pirro, M. Dohlus, S. Düsterer, H. T. Edwards, B. Faatz, A. A. Fateev, J. Feldhaus, K. Flöttmann, J. Frisch, L. Fröhlich, T. Garvey, U. Gensch, N. Golubeva, H.-J. Grabosch, B. Grigoryan, O. Grimm, U. Hahn, J.H. Han, M. v. Hartrott, K. Honkavaara, M. Hüning, R. Ischebeck, E. Jaeschke, M. Jablonka, R. Kammering, V. Katalev, B. Keitel S. Khodyachykh, Y. Kim, V. Kocharyan, M. Körfer, M. Kollewe, D. Kostin, D.
Krämer, M. Krassilnikov, G. Kube, L. Lilje, T. Limberg, D. Lipka, F. Löhl, M. Luong, C. Magne, J. Menzel, P. Michelato, V. Miltchev, M. Minty, W. D. Möller, L. Monaco, W. Müller, M. Nagl, O. Napoly, P. Nicolosi, D. Nölle, T. Nunez, A. Oppelt, C. Pagani, R. Paparella, B. Petersen, B. Petrosyan, J. Pflüger, P. Piot, E. Plönjes, L. Poletto, D. Proch, D. Pugachov, K. Rehlich, D. Richter, S. Riemann, M. Ross, J. Rossbach, M. Sachwitz, E. L. Saldin, W. Sandner, H. Schlarb, B. Schmidt, M. Schmitz, P. Schmüser, J.R. Schneider, E. A. Schneidmiller, H.-J. Schreiber, S. Schreiber, A. V. Shabunov, D. Sertore, S. Setzer, S. Simrock, E. Sombrowski, L. Staykov, B. Steffen, F. Stephan, F. Stulle, K. P. Sytchev, H. Thom, K. Tiedtke, M. Tischer, R. Treusch, D. Trines, I. Tsakov, A. Vardanyan, R. Wanzenberg, T. Weiland, H. Weise, M. Wendt, I. Will, A. Winter, K. Wittenburg, M. V. Yurkov, I. Zagorodnov, P. Zambolin, and K. Zapfe, Eur. Phys. J. D 37, 297 (2006).

[4] S. Schreiber, in Proceedings of the EPAC 2004 Conference (Lucerne, Switzerland), 2004, pp. 351-353.

[5] H. Weise, in Proceedings of the PAC 2003 Conference (Portland, OR, USA), 2003, pp. 673-677.

[6] S. Schreiber, in Proceedings of the FEL 2005 Conference (Stanford, CA, USA), 2005, pp. 545-548.

[7] K. Honkavaara, A. Brenger, R. Fischer, D. Nölle, K. Rehlich, L. Cacciotti, M. Castellano, G. DiPirro, M. Raparelli, R. Sorchetti, L. Catani, and A. Cianchi, in Proceedings of the PAC 2003 Conference (Portland, OR, USA), 2003, pp 2476-2478.

[8] A. Cianchi, L. Catani, E. Chiadroni, M. Raparelli, M. Castellano, G. Di Pirro, and K. Honkavaara, in Proceedings of the EPAC 2004 Conference (Lucerne, Switzerland), 2004, pp. 2619-2621.

[9] L. Catani, A. Cianchi, G. Di Pirro, and K. Honkavaara, Rev. Sci. Instrum. 76, 073303 (2005).

[10] M. Minty and F. Zimmermann, Measurements and Control of Charged Particle Beams (Springer, Berlin, 2003).

[11] J. Scheins, TESLA Report 2004-08, 2004.

[12] F. Löhl, DESY-THESIS 2005-014 and TESLA-FEL 200503 (2005).

[13] M. Krasilnikov, K. Abrahamyan, G. Asova, J. Bähr, G. Dimitrov, U. Gensch, H.-J. Grabosch, J. H. Han, D. Lipka, V. Miltchev, A. Oppelt, B. Petrosyan, D. Pose, S. Riemann, L. Staykov, F. Stephan, M. v. Hartrott, E. Jaeschke, D. Krämer, D. Richter, I. Bohnet, J.-P. Carneiro, K. Flöttmann, S. Schreiber, J. Rossbach, P. Michelato, L. Monaco, C. Pagani, D. Sertore, I. Tsakov, W. Sandner, I. Will, W. Ackermann, R. Cee, W. F. O. Müller, S. Setzer, and T. Weiland, in Proceedings of the EPAC 2004 Conference (Lucerne, Switzerland), 2004, pp. 360-362.

[14] K. Honkavaara, Y. Kim, and F. Löhl, in Proceedings of the DIPAC 2005 Conference (Lyon, France), 2005, pp. 81-83.

[15] K. Honkavaara, in Proceedings of the FEL 2005 Conference (Stanford, CA, USA), 2005, pp. 411-417.

[16] K. Honkavaara, F. Löhl, D. Nölle, S. Schreiber, E. Sombrowski, M. Sachwitz, M. Castellano, G. di Pirro, L. Catani, and A. Cianchi, in Proceedings of the FEL 2005 Conference (Stanford, CA, USA), 2005, pp. 122-125.

[17] Simulation by Y. Kim, DESY, 2005. 